\title{
Microsatellite instability screening in colorectal adenomas to detect Lynch syndrome patients? A systematic review and meta-analysis
}

\author{
Parag D. Dabir ${ }^{1,2} \cdot$ Carlijn E. Bruggeling ${ }^{1} \cdot$ Rachel S. van der Post ${ }^{1} \cdot$ Bas E. Dutilh ${ }^{3,4} \cdot$ Nicoline Hoogerbrugge $^{5} \cdot$ \\ Marjolijn J. L. Ligtenberg $\mathbb{D}^{1,5} \cdot$ Annemarie Boleij $^{1} \cdot$ Iris D. Nagtegaal $^{1}$
}

Received: 18 May 2019 / Revised: 1 October 2019 / Accepted: 13 October 2019 / Published online: 6 November 2019

(c) The Author(s), under exclusive licence to European Society of Human Genetics 2019

\begin{abstract}
The colorectal cancer spectrum has changed due to population screening programs, with a shift toward adenomas and early cancers. Whether it would be a feasible option to test these adenomas for detection of Lynch syndrome (LS) patients is unclear. Through meta-analysis and systematic review, risk factors for DNA mismatch repair deficiency (dMMR) and microsatellite instability (MSI) in adenomas were identified in LS and unselected patient cohorts. Data were extracted for patient age and MMR variant together with adenoma type, grade, size, and location. A total of 41 studies were included, and contained more than 519 LS patients and 1698 unselected patients with 1142 and 2213 adenomas respectively. dMMR/MSI was present in $69.5 \%$ of conventional adenomas in LS patients, compared with $2.8 \%$ in unselected patients. In the LS cohort, dMMR/MSI was more frequently present in patients older than 60 years (82\% versus $54 \%)$. dMMR/MSI was also more common in villous adenomas (84\%), adenomas over $1 \mathrm{~cm}(81 \%)$, and adenomas with high grade dysplasia (88\%). No significant differences were observed for dMMR/MSI in relation to MMR variants and location of adenomas. In the context of screening, we conclude that detection of dMMR/MSI in conventional adenomas of unselected patients is uncommon and might be considered as indication for LS testing. Within the LS cohort, $69.5 \%$ of LS patients could have been detected through dMMR/MSI screening of their conventional adenomas.
\end{abstract}

These authors contributed equally: Parag D. Dabir, Carlijn E. Bruggeling

Supplementary information The online version of this article (https:// doi.org/10.1038/s41431-019-0538-7) contains supplementary material, which is available to authorized users.

Iris D. Nagtegaal

Iris.Nagtegaal@radboudumc.nl

1 Department of Pathology, Radboud University Medical Center, Nijmegen, The Netherlands

2 Department of Pathology, Randers Regional Hospital, Randers, Denmark

3 Theoretical Biology and Bioinformatics, Utrecht University, Utrecht, The Netherlands

4 Centre for Molecular and Biomolecular Informatics, Radboud University Medical Center, Nijmegen, The Netherlands

5 Department of Genetics, Radboud University Medical Center, Nijmegen, The Netherlands

\section{Introduction}

Lynch syndrome (LS) is the most frequent cause of hereditary colorectal cancer (CRC). Patients with LS carry a germline variant affecting one of the proteins encoded by the DNA mismatch repair (MMR) genes MLH1, MSH2, MSH6, or PMS2 [1]. Loss of functional MMR proteins results in microsatellite instability (MSI) [2]. Patients with LS have an elevated risk to develop various types of cancers, of which the risk on endometrium cancer and CRC is remarkably high [3]. While traditionally clinical definitions were leading in the detection of LS patients, MMR/MSI testing on newly diagnosed CRC is increasingly advocated in order to identify these patients [4]. This identification is imperative for proper treatment and surveillance of individuals and their family members, thus preventing cancer development.

Population based screening for bowel cancer has been implemented in many countries to prevent $\mathrm{CRC}$, by detection and subsequent endoscopic removal of (advanced) adenomas [5]. Since MSI testing is currently only performed on CRC and not on precursor lesions, one could wonder whether detection of LS patients through population 
screening can be boosted by including adenomas. Identification of novel LS patients will facilitate early diagnosis and treatment of cancers in LS probands and their family.

If we assume that DNA mismatch repair deficiency (dMMR)/MSI is an early event in LS associated carcinogenesis, then dMMR/MSI testing of adenomas would be a useful strategy to detect LS. Multiple studies suggested that dMMR/MSI testing of adenomas has some value in diagnosing LS [6, 7], however, these studies are small and hardly reach consensus. Moreover, the overall incidence of dMMR/MSI in adenomas in the general (i.e., non-Lynch) population seems very low. To provide evidence for the use of adenomas in the detection of LS patients, we performed a meta-analysis in order to identify risk factors for dMMR/ MSI and to determine the feasibility to detect LS patients by testing those adenomas.

\section{Methods}

\section{Search strategy}

A comprehensive literature search for published studies was performed by using Pubmed and Embase (OvidSP software; Ovid Technologies, New York, NY) from inception to 7th of September 2018 using the following keywords: "(LS)" or "hereditary non-polyposis" or "mismatch repair" or "microsatellite instability" or "MSI" or "MMR protein" in combination with "polyp" or "adenoma" in combination with "colon" or "colonic" or "colorectal" (for the exact search terms see Supplementary Data 1). Additional searches were performed by manual cross-referencing.

Only original human studies published in English were selected by two independent reviewers (PDD, CEB) and cross checked by two reviewers (AB, IDN).

Reasons for exclusion included overlapping data, incomprehensive data, insufficient, and/or unclear data for analysis. In case of overlapping patient data, results of the largest study were included in the meta-analysis.

Patient groups from included studies were divided into "LS" and "Unselected patient" groups. The unselected patient group represents patients with adenomas with variable exclusion criteria for each study. Therefore, this patient group mainly represents sporadic cases, but importantly, may also include undiagnosed LS patients.

\section{Data extraction}

For each study, the number of patients and adenomas along with their MMR/MSI status tested by immunohistochemistry (IHC) and/or polymerase chain reaction (PCR) was obtained and combined with genetic information (germline testing or application of Bethesda or Amsterdam criteria).
Data on the method of MSI testing (IHC or PCR), number and type of markers, type of LS associated MMR variant (LS variant), age of the patient, adenoma type, grade, size, and location were extracted from all included studies. Data were independently extracted and checked by three reviewers (PDD, AB, and $\mathrm{CEB}$ ). Discrepancies after data check were reviewed by a fourth independent reviewer (IDN) in order to achieve consensus.

\section{Definitions}

We used the common term "MSI" instead of "MSI-High (MSI-H) by PCR" and "dMMR" for "deficient MMR staining by IHC" for readability. Because adenomas were often only tested by one method, we use the collective term "dMMR/MSI" for adenomas that classify for either or both statuses. All adenomas that were microsatellite stable or MSI-low were defined as "MSS" and adenomas that were MMR proficient were defined as "pMMR". For studies that used both methods, we included the most sensitive method i.e., the method in which most cases were dMMR/MSI. Cutoff points for age were set at 40 and 60 years; 40 years in order to select adenomas at an early age [8] and 60 years because it is the average age for the start of population screening programs $[5,9]$. To distinguish between smaller and larger adenomas, we used 5 and $10 \mathrm{~mm}$ as cut-off points. Proximal adenomas were located in the cecum, ascending colon, and transverse colon including splenic flexure and distal adenomas were located in descending colon, sigmoid, and rectum. All adenomas with a component of villousness were considered villous adenomas (including all tubulovillous adenomas). Dysplasia was divided into low and high grade, with moderate dysplasia included in low grade as per current nomenclature.

Serrated lesions were analyzed separately from conventional adenomas and included hyperplastic polyps, sessile serrated lesions, and traditional serrated adenomas [10].

\section{Statistical analysis}

A meta-analysis was performed to explore the potential of $\mathrm{dMMR} / \mathrm{MSI}$ in adenomas as a biomarker for LS syndrome. The sensitivity and specificity were calculated with a chisquare test. In addition, a two-tailed Mann-Whitney test was used to determine whether dMMR/MSI prevalence is different between LS adenomas and unselected adenomas. The Kruskal-Wallis test in combination with the Dunn's multiple comparison test was used to evaluate whether dMMR/MSI prevalence was different among different groups of adenomas; unselected adenomas and adenomas from specific LS variants; $M L H 1, M S H 2$, and MSH6. Each statistical test was performed using GraphPad Prism version 5.0 using a $95 \%$ CI. 
Table 1 Overview of the included studies

\begin{tabular}{|c|c|c|c|c|c|c|}
\hline First author & Ref. & $\begin{array}{l}\text { Origin } \\
\text { of cohort }\end{array}$ & $\begin{array}{l}\text { Germline }(G) \text { or } \\
\text { clinical definition }(C)\end{array}$ & $N$ (LS patients) & $N$ (Polyps) & $\begin{array}{l}\text { Technique PCR } \\
\text { (M) or IHC (I) }\end{array}$ \\
\hline Ahadova & 50 & Germany $^{a}$ & $\mathrm{G}$ & 15 & 21 & I \\
\hline Akiyama & 29 & Japan & $\mathrm{C}$ & 10 & 14 & M \\
\hline Aldecoa & 13 & Spain & $\mathrm{G}$ & 53 & 171 & I \\
\hline Chang & 51 & $\begin{array}{l}\text { USA } \\
\text { and Italy }\end{array}$ & G & 9 & 9 & I \\
\hline De Jong & 8 & Netherlands & $\mathrm{G}$ & 22 & 31 & I \\
\hline Ferreira & 30 & Portugal & $\mathrm{C}$ or $\mathrm{G}$ & 16 & 16 & M \\
\hline Giuffre & 31 & Germany & $\mathrm{C}$ & 17 & 18 & $\mathrm{M}$ and $\mathrm{I}$ \\
\hline Halvarsson & 32 & Sweden & $\mathrm{G}$ & 26 & 35 & $\mathrm{M}$ and $\mathrm{I}$ \\
\hline Iino & 33 & Australia & $\mathrm{C}$ or $\mathrm{G}$ & 24 & 47 & $\mathrm{M}$ and I \\
\hline Loukola & 34 & Finland & $\mathrm{C}$ or $\mathrm{G}$ & 12 & 12 & M \\
\hline Meijer & 25 & Netherlands & $\mathrm{G}$ & 38 & 50 & I \\
\hline Muller & 35 & Germany & $\mathrm{C}$ or $\mathrm{G}$ & 36 & 71 & $\mathrm{M}$ and $\mathrm{I}$ \\
\hline Pedroni & 36 & Italy & $\mathrm{C}$ or $\mathrm{G}$ & 7 & 12 & $\mathrm{M}$ and $\mathrm{I}$ \\
\hline Pino & 37 & USA & $\mathrm{G}$ & 15 & 44 & $\mathrm{M}$ and $\mathrm{I}$ \\
\hline Porkka & 23 & Finland & $\mathrm{G}$ & Unknown & 11 & M \\
\hline Rijcken & 56 & Netherlands & $\mathrm{C}$ or $\mathrm{G}$ & 40 & 90 & I \\
\hline Rijcken & 20 & Netherlands & $\mathrm{C}$ or $\mathrm{G}$ & Unknown & 25 & I \\
\hline Shia & 21 & USA & $\mathrm{C}$ or $\mathrm{G}^{\mathrm{c}}$ & Unknown & 29 & $\mathrm{M}$ and $\mathrm{I}$ \\
\hline Tanaka & 18 & Japan & $\mathrm{G}$ & 26 & 134 & I \\
\hline Valo & 22 & Finland & $\mathrm{G}$ & Unknown & 47 & $\mathrm{M}$ and $\mathrm{I}$ \\
\hline van Lier & 38 & Netherlands & $\mathrm{G}$ & 3 & 3 & $\mathrm{M}$ and $\mathrm{I}$ \\
\hline Velayos & 39 & USA & G & $3^{\mathrm{b}}$ & 4 & $\mathrm{M}$ and $\mathrm{I}$ \\
\hline Walsh & 40 & Australia & G & 69 & 109 & $\mathrm{M}$ and $\mathrm{I}$ \\
\hline Walsh & 41 & Australia & $\mathrm{C}$ or $\mathrm{G}$ & 7 & 22 & $\mathrm{M}$ and $\mathrm{I}$ \\
\hline Young & 42 & Australia & $\mathrm{C}$ or $\mathrm{G}$ & 18 & 25 & M \\
\hline Yurgelun & 43 & USA & $\mathrm{G}$ & 34 & 62 & $\mathrm{M}$ and $\mathrm{I}$ \\
\hline First author & Ref. & $\begin{array}{l}\text { Origin of } \\
\text { cohort }\end{array}$ & $\begin{array}{l}\text { Germline }(\mathrm{G}) \text { or } \\
\text { clinical definition }(\mathrm{C})\end{array}$ & $\begin{array}{l}N \text { (Unselected } \\
\text { patients) }\end{array}$ & $N$ (Polyps) & $\begin{array}{l}\text { Technique PCR } \\
\text { (M) or IHC (I) }\end{array}$ \\
\hline Basterra & 44 & Spain & No & 187 & 187 & I \\
\hline Ferreira & 30 & Portugal & No & 23 & 27 & $\mathrm{M}$ \\
\hline Goverde & 54 & Netherlands & No & 400 & 400 & I \\
\hline Koh & 45 & USA & No & $23^{\mathrm{b}}$ & 38 & $\mathrm{M}$ and $\mathrm{I}$ \\
\hline Kushnir & 46 & USA & No & 66 & 66 & I \\
\hline Loukola & 34 & Finland & No & 367 & 390 & $\mathrm{M}$ \\
\hline Meijer & 25 & Netherlands & No & Unknown & 19 & I \\
\hline $\begin{array}{l}\text { Mendel- } \\
\text { sohn }\end{array}$ & 7 & USA & No & 208 & 259 & I \\
\hline Patil & 26 & USA & No & Unknown & 127 & $\mathrm{M}$ and $\mathrm{I}$ \\
\hline Pedroni & 36 & Italy & No & 3 & 6 & $\mathrm{M}$ and $\mathrm{I}$ \\
\hline Reveron & 27 & USA & No & Unknown & 27 & I \\
\hline $\begin{array}{l}\text { Ricciar- } \\
\text { diello }\end{array}$ & 47 & Italy & No & 70 & 70 & $\mathrm{M}$ and $\mathrm{I}$ \\
\hline Samowitz & 9 & USA & No & 48 & 93 & $\mathrm{M}$ \\
\hline Samowitz & 24 & USA & No & Unknown & 71 & M \\
\hline Sekine & 17 & Japan & No & 25 & 84 & $\mathrm{M}$ and $\mathrm{I}$ \\
\hline
\end{tabular}


Table 1 (continued)

\begin{tabular}{|c|c|c|c|c|c|c|}
\hline First author & Ref. & $\begin{array}{l}\text { Origin of } \\
\text { cohort }\end{array}$ & $\begin{array}{l}\text { Germline }(\mathrm{G}) \text { or } \\
\text { clinical definition }(\mathrm{C})\end{array}$ & $\begin{array}{l}N \text { (Unselected } \\
\text { patients) }\end{array}$ & $N$ (Polyps) & $\begin{array}{l}\text { Technique PCR } \\
\text { (M) or IHC (I) }\end{array}$ \\
\hline Sheridan & 53 & USA & No & 6 & 6 & I \\
\hline Strul & 48 & Israel & No & 40 & 40 & M \\
\hline Ueda & 49 & Japan & No & 76 & 76 & M \\
\hline van Lier & 38 & Netherlands & No & 122 & 122 & $\mathrm{M}$ and $\mathrm{I}$ \\
\hline Velayos & 39 & USA & No & $34^{\mathrm{b}}$ & 40 & $\mathrm{M}$ and I \\
\hline Yamane & 28 & Brazil & No & 103 & 103 & M \\
\hline
\end{tabular}

${ }^{\mathrm{a}}$ Unclear

${ }^{\mathrm{b}}$ Only patients under the age of 40 years are included

Including Lynch like patients

To determine whether dMMR/MSI is a predominant feature in distinct categories of LS adenomas, an additional meta-analysis was performed using age, location, type, size, and grade as stratification factors (see "Definitions"). Also output risk ratios (RR) with 95\% confidence interval (CI) were calculated. Forest plots were generated to demonstrate consistency of the results. Forest plots for various parameters were obtained and compared with dMMR/MSI status.

To determine effect size, Z-statistic was calculated (standardized mean difference). Heterogeneity was assessed using a chi-square test for heterogeneity with a $P$ value of $<0.10$ to show the presence of significant heterogeneity. Furthermore, we applied $I^{2}$ statistics (percentage of variation across studies that is a result of heterogeneity rather than chance) in combination with Tau-squared estimate of between-study variance in a random effect meta-analysis. Forest plot analysis was performed with Review Manager (version 5.3; Cochrane Tech, London, United Kingdom).

\section{Quality assessment and risk of bias}

A scale to assess the quality of the reporting of the studies was developed based on the REMARK guidelines, focusing on dMMR/MSI, and adenomas (Supplementary Table 1) $[11,12]$. All included studies were subjected to quality assessment. The association between the quality of reporting and the Hazard Ratio was analyzed with scatter plots and nonparametric correlation testing. Publication bias was assessed by symmetry in funnel plots. These data were generated using Review Manager (version 5.3; Cochrane Tech, London, United Kingdom).

\section{dMMR/MSI status consistency analysis}

To statistically test if dMMR/MSI status tends to be consistent within patients, a permutation analysis was performed, in which dMMR/MSI status was randomly shuffled 10,000 times across all adenomas, and the percentage of
dMMR/MSI status-consistent patients was assessed (the results are consistent if adenomas are annotated as dMMR/ MSI positive with $p=0.68$ ). This randomization analysis yields an expected frequency that patients have a consistent dMMR/MSI status, given the distribution of the number of adenomas analyzed per patient and the percentage of dMMR/MSI positive adenomas. These permutation simulations allow us to calculate an enrichment score to assess how over- or underrepresented the observed number of patients with a consistent dMMR/MSI status is, as the $\log _{2}$ (observed/expected).

\section{Results}

\section{Search results}

A total of 919 studies were identified: 447 studies by the Pubmed database search and 472 by using Embase. Duplicates were excluded $(n=153)$. Another 722 studies were excluded because they did not meet the general inclusion criteria (Supplementary Fig. 1). We included one conference abstract, since the data were extensively described [13]. Another three studies were excluded because of unclear or insufficient data $[14,15]$ or because of overlapping data [16]. For one cohort, we used data described in two separate papers [17, 18].

Out of the remaining 41 studies selected for this review, 20 studies exclusively tested LS patients, 15 studies tested unselected patients, and six studies contained both patient groups (Table 1). The LS group is diverse, since some studies included only patients with a proven LS variant, while others fulfilled either Amsterdam criteria or Bethesda guidelines [19]. Not all studies were clear on the exact number of patients and adenomas included in their cohorts. We included at least 519 LS patients (unknown numbers of patients in three studies) [20-23] with 1142 polyps of which 883 were conventional adenomas. For the unselected 
cohort, we included at least 1698 patients (unknown numbers of patients in five studies [24-28]), with 2213 adenomas of which 2119 were conventional adenomas.

\section{Quality of the studies}

Studies were subjected to quality assessment (Supplementary Table 1). Out of 41 studies used for meta-analysis, 36 studies scored $\geq 50 \%$ of quality criteria which could be used for correlation of dMMR/MSI with colorectal adenoma characteristics. The mean percentage of items reported in studies with data for correlation was $67.9 \%$ (95\% CI range 60.5-75.3).

\section{Testing methods}

Two different approaches were used to identify LS in adenomas (Supplementary Table 2); dMMR by IHC or MSI by PCR. In 18 studies covering 366 adenomas that applied both techniques, the discordance varied from $1 \%$ in the unselected group to $15 \%$ in the LS cohorts. dMMR without MSI was more common than the other way around, but only occurred in the LS cohort (32 adenomas). MSI without dMMR, occurred in three adenomas of the unselected cohort versus 14 in the LS cohort.

\section{Prevalence of MSI}

dMMR/MSI was present in $69.5 \%$ of the conventional adenomas (614/883) of LS patients [8, 13, 18, 20-22, 25, 29-43], compared with $2.8 \%(60 / 2119)$ from the unselected patients $[7,9,17,24-26,30,34,36,38,39,44-49]$ when grouping all studies. Alternatively, the mean percentage of dMMR/MSI was $64.9 \%$ (95\% CI 55.8-76.01) amongst LS cohorts and $4.0 \%$ (95\% CI 1.1-7.0) amongst unselected patient cohorts when analyzing each study individually (significant difference with $p<0.0001$ ) (Fig. 2a) $[8,18,20-22,25,32,36-$ $38,40,41,43]$. Using dMMR/MSI status of adenomas as a diagnostic marker for LS resulted in a sensitivity of $69.5 \%$ (95\% CI 66.4-72.6) and a specificity of $97.2 \%$ (95\% CI 96.4-97.8). Sensitivity increased to $71.7 \%$ (95\% CI 68.1-75.0), when restricted to LS patients with a proven LS variant (Fig. 1), which was not significantly higher compared to the overall LS patient group $(p=0.19)$.

\section{Relation to LS variants}

We analyzed the prevalence of dMMR/MSI in adenomas of LS patients per MMR gene affected (Fig. 1). The mean percentage of dMMR/MSI was $79.9 \%$ versus $73.4 \%$ versus $52.8 \%$ for $M L H 1, M S H 2$, and $M S H 6$, respectively $[8,18,20-23,25,32,36-38,40,41,43,50,51]$. According to the Dunn's multiple comparison test, all three LS variants

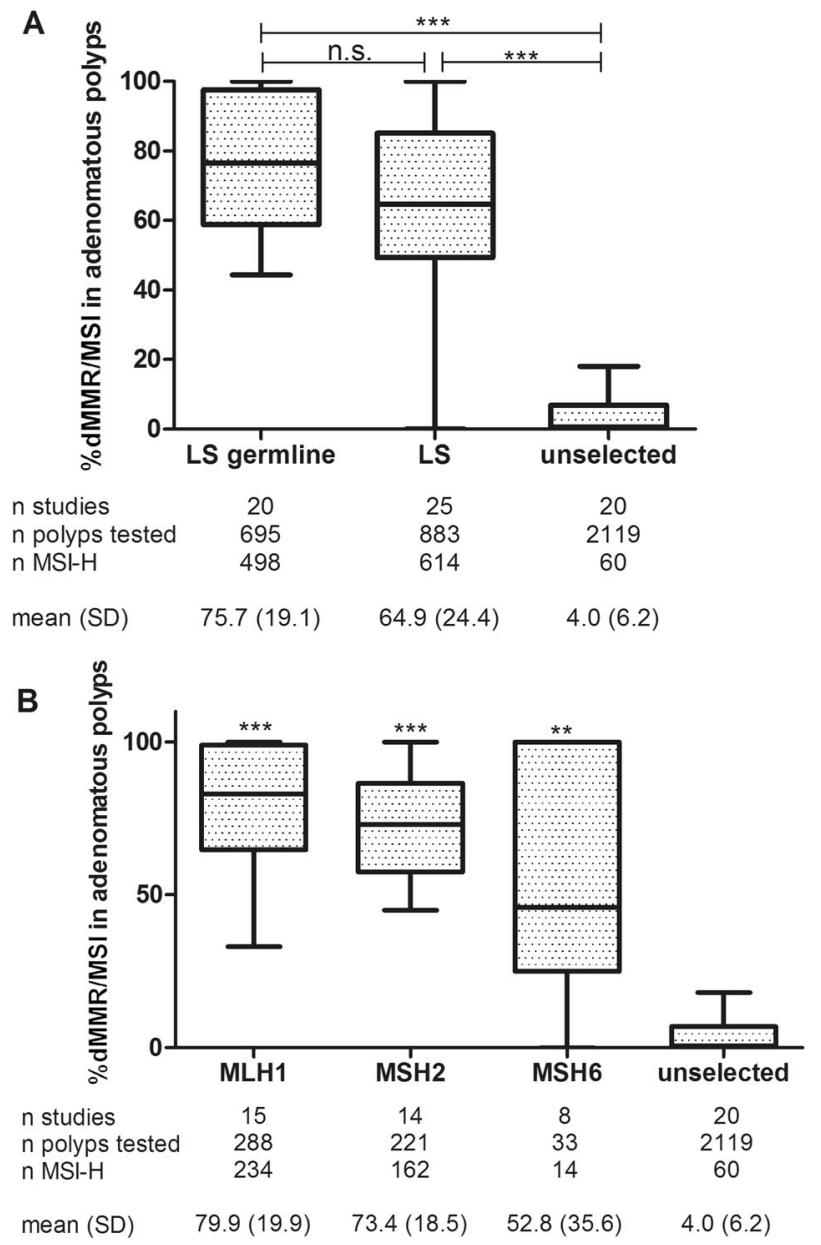

Fig. 1 Microsatellite instability in adenomas. a Percentage of dMMR/ MSI in adenomas of germline confirmed LS, all LS patients and unselected patients. b Impact of specific germline variants on the presence of dMMR/MSI $(* * * p<0.001, * * p<0.01)$

groups displayed significantly higher prevalence compared with the unselected group (MLH1; $p<0.001, M S H 2 ; p<$ 0.001 , and MSH6; $p<0.05$ ), however differences between the genes were not significant. Only two studies included patients with PMS2 LS variants [13, 40], in which two adenomas showed dMMR/MSI [40].

\section{Relation to age}

In the LS group, $54.5 \%$ of the adenomas of patients under the age of 60 years showed dMMR/MSI compared with $82.3 \%$ in the group with older patients (RR $0.8,95 \%$ CI 0.7-0.95) (Fig. 2, Supplementary Fig. 2A) [8, 18, 29, 31$33,37,41,43]$. No significant heterogeneity was observed $\left(I^{2}=25 \%\right)$. Changing of the cut-off levels to 40 years did not result in a significant difference (RR 1.2, 95\% CI 0.9-1.4) (Supplementary Fig. 2B).

In the unselected patient group, 8 out of 47 adenomas showed $\mathrm{dMMR} / \mathrm{MSI}$ in the age group $40-60$ years. This 


\begin{tabular}{lrl} 
Category & dMMR/MSI \% & RR $(95 \% \mathrm{CI})$ \\
\hline$<60 \mathrm{y} / \geq 60 \mathrm{y}$ & $55 \% / 82 \%$ & $0.80(0.67,0.95)$ \\
proximal / distal & $73 \% / 66 \%$ & $1.08(0.91,1.27)$ \\
tubular / villous & $60 \% / 84 \%$ & $0.81(0.71,0.93)$ \\
$<10 \mathrm{~mm} / \geq 10 \mathrm{~mm}$ & $60 \% / 81 \%$ & $0.73(0.58,0.92)$ \\
LGD / HGD & $66 \% / 88 \%$ & $0.72(0.62,0.84)$
\end{tabular}
0.1
Fig. 2 Summary of forest plots of relative risk of dMMR/MSI in adenomas of LS patients. LS adenomas were split into two groups according to the following categories: age of patients $(<60$ year or $>60$ year), adenoma location (proximal or distal), type (tubular or villous),

frequency was only 1 of 144 adenomas in patients under 40 years old versus none out of 90 in the group older than 60 years [39, 45, 46, 49, 52, 53]. Additional information about age was limited.

\section{Location of adenomas}

In the LS group, there was no significant difference in $\mathrm{dMMR} / \mathrm{MSI}$ positivity in relation to location, with $72 \%$ of the proximal adenomas and $66 \%$ of the distal adenomas showing dMMR/MSI (RR 1.1, 95\% CI 0.9-1.3) (Fig. 2, Supplementary Fig. 2C) [8, 18, 20, 25, 29, 32, 33, 37, 39, 40, 43]. Moderate heterogeneity was observed $\left(I^{2}=46 \%\right)$, which was mainly caused by a difference in detection method (IHC; $I^{2}=35 \%$ and PCR; $I^{2}=67 \%$ ).

In the unselected group, $11 \%$ of the proximal adenomas and $5 \%$ of the distal adenomas, dMMR/MSI was observed (RR 2.4, 95\% CI 1.2-4.6) without heterogeneity $\left(I^{2}=0 \%\right)$ [9, 17, 24, 44, 47, 49, 52].

\section{Type of adenomas}

In the LS group, there was a significant difference between prevalence of $\mathrm{dMMR} / \mathrm{MSI}$ in villous adenomas $(84 \%)$ compared with tubular adenomas $(60 \%)$ (Fig. 2, Supplementary Fig. 2) (RR 0.8, 95\% CI 0.7-0.9). No heterogeneity was observed $\left(I^{2}=0 \%\right)[8,25,29,31,32,37-40]$.

In the unselected group, there were only three studies from which this data could be extracted [30, 45, 54], but all tubular $(n=253)$ and almost all villous adenomas (196 out of 197) were MSS.

\section{Size of adenomas}

In the LS group, a cut-off of $10 \mathrm{~mm}$ resulted in a significant difference; $60.2 \%$ of the adenomas $<10 \mathrm{~mm}$ showed size $(<10 \mathrm{~mm}$ or $>10 \mathrm{~mm}$ ), grade (low grade dysplasia (LGD), or high grade dysplasia (HGD)). The percentage of dMMR/MSI for per category was calculated. Differences were expressed as risk ratio (RR). The summary is based on Supplementary Fig. $2 \mathrm{~A}-\mathrm{G}$

dMMR/MSI compared with $81.4 \%$ of the adenomas $>10 \mathrm{~mm}$ (RR 0.73, 95\% CI 0.6-0.9) (Fig. 2, Supplementary Fig. 2F) $[31-33,37,38,40,43,51]$. A $5 \mathrm{~mm}$ cut-off resulted in a difference of $49.2 \%$ versus $70.0 \%$, respectively, but this was not significant (Supplementary Fig. 2E).

In the unselected group, there was no difference between the smaller and larger adenomas (cut-off of $5 \mathrm{~mm}$ : RR 0.4, 95\% CI $0.1-1.1$, cut-off of $10 \mathrm{~mm}$ : RR $0.4,95 \% \mathrm{CI}$ $0.1-0.9)$ [17, 24, 44, 49].

\section{Grade of adenomas}

In the LS group, dMMR/MSI was present in $87.7 \%$ in adenomas with high grade dysplasia compared with $65.7 \%$ in adenomas with low grade dysplasia (RR 0.72, 95\% CI 0.62-0.84) (Fig. 2, Supplementary Fig. 2G) [8, 18, 20, 22, 25, 29-33, 35, 37, 40, 50, 51]. Substantial heterogeneity was observed $\left(I^{2}=59 \%\right)$.

In the unselected group, $5 \%$ of adenomas with high grade dysplasia (11 out of 212) showed dMMR/MSI; while $8 \%$ of adenomas with low grade dysplasia (10 out of 130) showed dMMR/MSI [27, 30, 39, 44, 49, 53, 55].

\section{dMMR/MSI status consistency analysis}

To investigate the attributed value of testing a second or third polyp, we first tested whether dMMR/MSI status is consistent in adenomas of LS patients. $182 \mathrm{LS}$ patients of whom 131 were genetically confirmed to have a LS variant were included in this analysis (see Supplementary Fig. 4) [8, 17, 29-32, 34, 36, 37, 39, 41-43]. A randomization test was performed, in which dMMR/MSI status of adenomas was permuted, allowing to calculate an observed/expected ratio of $\mathrm{dMMR} / \mathrm{MSI}$ consistency in patients. As shown in Supplementary Fig. 5A \& B, this analysis revealed that patients tend to have a consistent dMMR/MSI status: there 
are more patients with consistent adenomas and fewer patients with inconsistent adenomas than expected. All raw data is available in Supplementary File 1. In addition, we performed a second analysis, in which we evaluate the chance of finding a dMMR/MSI adenoma, after the first adenoma is tested to be pMMR/MSS. 54 germline confirmed LS patients were included in this analysis. $62 \%$ of LS patients that had multiple adenomas tested, had an adenoma with dMMR/MSI status, after the first adenoma was tested to be pMMR/MSS. This suggests that testing more than one adenoma per patient decreases the risk of missing LS patients.

\section{Serrated polyps}

Information about dMMR/MSI status in serrated polyps was limited. Hyperplastic polyps in LS patients showed variable results, 4 [41, 43] out of 333 [13, 32, 33, 40, 42, 51, 56] hyperplastic polyps showed dMMR/MSI. Hyperplastic polyps were described in only one of the unselected group and none of 38 hyperplastic polyps displayed dMMR/MSI [28].

Traditional serrated adenomas in LS patients showed dMMR/MSI in 9 out of 12 [8, 32, 40-42]. Sessile serrated lesions only displayed dMMR/MSI in 1 out of 19 in the LS cohort [13, 40-43, 56]. However, no dysplasia was reported in the LS cohort. In the unselected cohort, 4 out of 6 sessile serrated lesions with dysplasia showed dMMR/MSI and MLH1 loss [53]. In the unselected group one study reported no cases of dMMR/MSI in 13 sessile serrated lesions and two traditional serrated adenomas, but dysplasia was not reported in this study [28].

Forest plot analysis was repeated by including serrated lesions along with adenomas which did not change the initial outcomes.

\section{Discussion}

In the current study we have shown that dMMR/MSI is a rare finding in conventional adenomas of unselected patients $(2.8 \%)$, but can be identified in the majority of adenomas from patients with LS (69.5\%). dMMR/MSI adenomas are highly indicative of LS with a specificity of 97.2\%. Notably, some dMMR/MSI cases in the unselected cohort may be as yet undetected LS patients, because not all cases were followed-up with genetic testing. This is also suggested in some of the included papers [26, 34, 44, 48] and suggests that our calculation for specificity is a modest underestimation. While dMMR/MSI in adenomas is a powerful LS marker, its utility in detecting novel patients is limited by the absence of MSI in $30.5 \%$ of adenomas in LS patients in combination with the relative low frequency of undetected LS patients.
When performing dMMR/MSI testing on adenomas, it is important to understand that the properties of this marker are different than in CRC. First of all, dMMR/MSI is present in $96 \%$ cases of LS associated CRC [57]. Therefore, expanding dMMR/MSI testing to include adenomas will most likely result in decreased sensitivity of testing. However, by expanding dMMR/MSI testing to adenomas, overall testing results will be more specific. This is because sporadic dMMR/MSI in adenomas is rare, while $\sim 15 \%$ of sporadic CRC displays dMMR/MSI [58]. In other words, $\mathrm{dMMR} / \mathrm{MSI}$ testing in adenomas is less sensitive, but once dMMR/MSI is detected, the chance that this case is LS patient will be higher.

Both the increased specificity and decreased sensitivity of dMMR/MSI as a marker for LS in adenomas suggest that dMMR/MSI is a late event occurring in already established adenomas. This is supported by the presence of somatic variants of $A P C, C T N B B 1$, and $T P 53[17,50,51]$ in LSassociated adenomas. However, alternative pathways, albeit less common, are likely present in a subset of LS-associated carcinomas, with their origin in MMR deficient crypts, bypassing adenomatous precursor lesions [50]. These lesions are obviously not included in our meta-analysis.

The most common underlying mechanism for dMMR/ MSI in sporadic CRC is hypermethylation of MLH1 promoter region, which contributes to up to $60 \%$ of all dMMR/ MSI cases [59, 60]. In sessile serrated lesions dMMR/MSI frequently co-occurs with variants in the $B R A F$ oncogene [61]. While in our cohort few sessile serrated lesions with dysplasia were included, 4 out of 6 showed loss of MLH1, in line with current literature. Among LS patients, serrated polyps are relatively uncommon [62]. The low prevalence of serrated polyps in LS patients in combination with the high frequency of dMMR/MSI among sessile serrated lesions within the sporadic cohort, suggests that testing these polyps will be an inefficient strategy to identify LS patients.

Following up on the observation that dMMR/MSI is infrequent in adenomas, we explored whether dMMR/MSI is more frequent in certain types of adenomas through a subgroup analysis. To boost the power of the analysis, all LS patients were included in the analysis, irrespective of germline testing. In the era of population screening for bowel cancer, the focus is on advanced adenomas, that are defined as adenomas $>10 \mathrm{~mm}$ and/or with a villous component and/ or high grade dysplasia. These advanced adenomas are considered immediate precursors of CRC and their removal will ultimately result in decreased rates of cancer in populations where screening is implemented. The results from CRC population screening showed that in $3.9-41 \%$ of performed colonoscopies advanced adenomas were found and $2.2-10.1 \%$ CRC [5]. In order not to miss out on detection of LS patients, we explored the possibilities 
in this particular subgroup of adenomas. Due to limited information, only one discriminating factor could be used at the same time to differentiate between subgroups. Forest plot analysis revealed that characteristics of advanced adenomas significantly increase dMMR/MSI prevalence in LS patients from $60-65 \%$ to $81-88 \%$. These results further support the hypothesis that $\mathrm{dMMR} / \mathrm{MSI}$ is frequently a later event in LS carcinogenesis and also suggests that relatively more LS patients will be detected through focusing the screening of advanced adenomas.

Since current guidelines generally accept CRC at young age [8] and right-sided CRC as a risk factor for LS [19], we hypothesized that both young age and proximal location would increase the detection rate of dMMR/MSI in adenomas. However, there was no difference between leftsided and right-sided adenomas, and no increased dMMR/ MSI rates in younger patients. Instead, older age significantly aligned with higher dMMR/MSI prevalence, which suggests an increased risk of second hit affecting MMR expression in older patients [63]. Thus, patient age and adenoma location are not helpful factors to boost the sensitivity of dMMR/MSI as a marker for LS.

Based on the NICE report (2017) [64], the dMMR/MSI testing of CRC cases to detect LS cases is considered costeffective. Based on a prevalence of $2.8 \%$ dMMR/MSI in adenomas of unselected patients, we conclude that 36 adenomas should be tested, to detect one adenoma that shows dMMR/MSI. This should be followed up by further tests in order to determine the patients' risk on LS [34, 38]. While this detection rate is considered cost-effective in CRC [4], it may be different for adenomas. More patients present with adenomas than CRC and also the sensitivity of testing will be lower. Importantly, our results suggest that this can be partly overcome by focusing on advanced adenomas. We found that LS adenomas with characteristics of advanced stage, display dMMR/MSI in $81-88 \%$ cases. In addition, inclusion of adenomas or advanced adenomas in screening will presumably have a large effect on costs, given the fact that we identify the index patients before they have developed cancer. Hence, a study of cost-effectiveness of including adenomas in dMMR/MSI screening is required to understand its putative impact.

Taken together, it appears that dMMR/MSI is associated with advanced stages of conventional adenomas, resulting in decreased sensitivity of this biomarker for LS when it is applied to adenomas instead of carcinomas. By focusing on advanced adenomas, this reduced sensitivity may only be partly overcome. While dMMR/MSI in adenomas remains a specific marker for LS, the use of dMMR/MSI as a screening tool will leave a substantial portion of LS patients undiagnosed. To summarize, this meta-analysis has elucidated the value of dMMR/MSI testing on adenomas. In addition, we have evaluated the prevalence of patients that may be at risk for LS within an unselected cohort. This data may serve as a foundation for future cost-effectiveness studies.

\section{Data availability}

Most data generated or analyzed during this study are included in this published article and its supplementary data. Additional information files are available from the corresponding author upon reasonable request.

Funding $\mathrm{AB}$ is supported by Netherlands Organization for Scientific Research (NWO) Veni grant 016.166.089. CEB and AB are supported by the Dutch cancer society (KWF kankerbestrijding) KUN 20157739. BED is supported by Netherlands Organization for Scientific Research (NWO) Vidi grant 864.14.004.

Author contributions PDD, CEB, BED, AB, and IDN contributed to study design, data-acquisition, -analysis, and -interpretation, as well as drafting of the paper. $\mathrm{AB}$ and IDN supervised the project. All authors performed critical revision of the paper for important intellectual content.

\section{Compliance with ethical standards}

Conflict of interest The authors declare that they have no conflict of interest.

Ethical approval No ethical review board was involved in this metaanalysis, because public documents are subjects of the study. Data was collected through an unbiased search strategy with well-defined inclusion and exclusion criteria. Data was extracted, analyzed accurately and was tested for bias [65].

Publisher's note Springer Nature remains neutral with regard to jurisdictional claims in published maps and institutional affiliations.

\section{References}

1. WCoTEB. Digestive system tumours, WHO classification of tumours. 5th ed (World Health Organization, Genève, 2019) $1: 635$.

2. Lynch HT, Snyder CL, Shaw TG, Heinen CD, Hitchins MP. Milestones of Lynch syndrome: 1895-2015. Nat Rev Cancer. 2015;15:181-94.

3. Moller P, Seppala T, Bernstein I, Holinski-Feder E, Sala P, Evans DG, et al. Cancer incidence and survival in Lynch syndrome patients receiving colonoscopic and gynaecological surveillance: first report from the prospective Lynch syndrome database. Gut. 2017;66:464-72.

4. Leenen $\mathrm{CH}$, Goverde A, de Bekker-Grob EW, Wagner A, van Lier MG, Spaander MC, et al. Cost-effectiveness of routine screening for Lynch syndrome in colorectal cancer patients up to 70 years of age. Genet Med. 2016;18:966-73.

5. Navarro M, Nicolas A, Ferrandez A, Lanas A. Colorectal cancer population screening programs worldwide in 2016: an update. World J Gastroenterol. 2017;23:3632-42.

6. de la Chapelle A, Hampel H. Clinical relevance of microsatellite instability in colorectal cancer. J Clin Oncol. 2010;28:3380-7. 
7. Mendelsohn RB, Herzog K, Shia J, Rahaman N, Stadler ZK, Shike M. Molecular screening for lynch syndrome in young patients with colorectal adenomas. Clin Colorectal Cancer. 2017;16:173-7.

8. De Jong AE, Morreau H, Van Puijenbroek M, Eilers PH, Wijnen J, Nagengast FM, et al. The role of mismatch repair gene defects in the development of adenomas in patients with HNPCC. Gastroenterology. 2004;126:42-8.

9. Samowitz WaS, M. Microsatellite instability in colorectal adenomas. Gastroenterol. 1997;112:1515-9.

10. Bateman AC, Shepherd NA. UK guidance for the pathological reporting of serrated lesions of the colorectum. J Clin Pathol. 2015;68:585-91.

11. Knijn N, Simmer F, Nagtegaal ID. Recommendations for reporting histopathology studies: a proposal. Virchows Arch. 2015;466:611-5.

12. McShane LM, Altman DG, Sauerbrei W, Taube SE, Gion M, Clark GM, et al. Reporting recommendations for tumor marker prognostic studies (REMARK). J Natl Cancer Inst. 2005; 97:1180-4.

13. Aldecoa I, Rakislova N, Carballal S, Montironi C, Herrero L, Sanchez A, et al. Large size and high grade dysplasia are strong predictors of mismatch repair deficiency in lynch syndromeassociated colorectal adenomas. Lab Investig. 2016;96:160A-1A.

14. Bacher JW, Sievers CK, Albrecht DM, Grimes IC, Weiss JM, Matkowskyj KA, et al. Improved Detection of microsatellite instability in early colorectal lesions. PLoS One. 2015;10: e0132727.

15. Balbinotti RA, Ribeiro U Jr., Sakai P, Safatle-Ribeiro AV, Balbinotti SS, Scapulatempo C, et al. hMLH1, hMSH2 and cyclooxygenase-2 (cox-2) in sporadic colorectal polyps. Anticancer Res. 2007;27(6c):4465-71.

16. Ferreira AM, Westers H, Sousa S, Wu Y, Niessen RC, OlderodeBerends $\mathrm{M}$, et al. Mononucleotide precedes dinucleotide repeat instability during colorectal tumour development in Lynch syndrome patients. J Pathol. 2009;219:96-102.

17. Sekine S, Mori T, Ogawa R, Tanaka M, Yoshida H, Taniguchi H, et al. Mismatch repair deficiency commonly precedes adenoma formation in lynch syndrome-associated colorectal tumorigenesis. Mod Pathol. 2017;1-8.

18. Tanaka M, Nakajima T, Sugano K, Yoshida T, Taniguchi H, Kanemitsu Y, et al. Mismatch repair deficiency in Lynch syndrome-associated colorectal adenomas is more prevalent in older patients. Histopathology. 2016;69:322-8.

19. Richman S. Deficient mismatch repair: read all about it (Review). Int J Oncol. 2015;47:1189-202.

20. Rijcken FE, Hollema H, Kleibeuker JH. Proximal adenomas in hereditary non-polyposis colorectal cancer are prone to rapid malignant transformation. Gut. 2002;50:382-6.

21. Shia J, Klimstra DS, Nafa K, Offit K, Guillem JG, Markowitz AJ, et al. Value of immunohistochemical detection of DNA mismatch repair proteins in predicting germline mutation in hereditary colorectal neoplasms. Am J Surg Pathol. 2005;29:96-104.

22. Valo S, Kaur S, Ristimaki A, Renkonen-Sinisalo L, Jarvinen H, Mecklin JP, et al. DNA hypermethylation appears early and shows increased frequency with dysplasia in Lynch syndrome-associated colorectal adenomas and carcinomas. Clin Epigenetics. 2015;7:71.

23. Porkka N, Valo S, Nieminen TT, Olkinuora A, Maki-Nevala S, Eldfors S, et al. Sequencing of Lynch syndrome tumors reveals the importance of epigenetic alterations. Oncotarget. 2017;8: 108020-30.

24. Samowitz WS, Slattery ML. Transforming growth factor-beta receptor type 2 mutations and microsatellite instability in sporadic colorectal adenomas and carcinomas. Am J Pathol. 1997;151:33-5.

25. Meijer TW, Hoogerbrugge N, Nagengast FM, Ligtenberg MJ, van Krieken JH. In Lynch syndrome adenomas, loss of mismatch repair proteins is related to an enhanced lymphocytic response. Histopathology. 2009;55:414-22.

26. Patil DT, Bronner MP, Portier BP, Fraser CR, Plesec TP, Liu X. A five-marker panel in a multiplex PCR accurately detects microsatellite instability-high colorectal tumors without control DNA. Diagn Mol Pathol. 2012;21:127-33. part B

27. Reveron D, Lopez C, Gutierrez S, Sayegh ZE, Antonia T, Dutil J, et al. Frequency of mismatch repair protein deficiency in a Puerto Rican population with colonic adenoma and adenocarcinoma. Cancer Genom Proteom. 2018;15:265-71.

28. Yamane LS, Scapulatempo-Neto C, Alvarenga L, Oliveira CZ, Berardinelli GN, Almodova E, et al. KRAS and BRAF mutations and MSI status in precursor lesions of colorectal cancer detected by colonoscopy. Oncol Rep. 2014;32:1419-26.

29. Akiyama Y, Nagasaki H, Yagi KO, Nomizu T, Yuasa Y. Betacatenin and adenomatous polyposis coli (APC) mutations in adenomas from hereditary non-polyposis colorectal cancer patients. Cancer Lett. 2000;157:185-91.

30. Ferreira S, Claro I, Lage P, Filipe B, Fonseca R, Sousa R, et al. Colorectal adenomas in young patients: microsatellite instability is not a useful marker to detect new cases of Lynch syndrome. Dis Colon Rectum. 2008;51:909-15.

31. Giuffre G, Muller A, Brodegger T, Bocker-Edmonston T, Gebert $\mathrm{J}$, Kloor M, et al. Microsatellite analysis of hereditary nonpolyposis colorectal cancer-associated colorectal adenomas by laser-assisted microdissection: correlation with mismatch repair protein expression provides new insights in early steps of tumorigenesis. J Mol Diagn. 2005;7:160-70.

32. Halvarsson B, Lindblom A, Johansson L, Lagerstedt K, Nilbert $\mathrm{M}$. Loss of mismatch repair protein immunostaining in colorectal adenomas from patients with hereditary nonpolyposis colorectal cancer. Mod Pathol. 2005;18:1095-101.

33. Iino H, Simms L, Young J, Arnold J, Winship IM, Webb SI, et al. DNA microsatellite instability and mismatch repair protein loss in adenomas presenting in hereditary non-polyposis colorectal cancer. Gut. 2000;47:37-42.

34. Loukola A, Salovaara R, Kristo P, Moisio AL, Kaariainen H, Ahtola $\mathrm{H}$, et al. Microsatellite instability in adenomas as a marker for hereditary nonpolyposis colorectal cancer. Am J Pathol. 1999;155:1849-53.

35. Muller A, Beckmann C, Westphal G, Bocker Edmonston T, Friedrichs N, Dietmaier W, et al. Prevalence of the mismatchrepair-deficient phenotype in colonic adenomas arising in HNPCC patients: results of a 5-year follow-up study. Int J Colorectal Dis. 2006;21:632-41.

36. Pedroni M, Sala E, Scarselli A, Borghi F, Menigatti M, Benatti P, et al. Microsatellite instability and mismatch-repair protein expression in hereditary and sporadic colorectal carcinogenesis. Cancer Res. 2001;61:896-9.

37. Pino MS, Mino-Kenudson M, Wildemore BM, Ganguly A, Batten $\mathrm{J}$, Sperduti I, et al. Deficient DNA mismatch repair is common in Lynch syndrome-associated colorectal adenomas. J Mol Diagn. 2009;11:238-47.

38. Van Lier MGF, Leenen CHM, Wagner A, Ramsoekh D, Dubbink HJ, Van Den Ouweland AMW, et al. Yield of routine molecular analyses in colorectal cancer patients $<70$ years to detect underlying lynch syndrome. J Pathol. 2012;226: 764-74.

39. Velayos FS, Allen BA, Conrad PG, Gum J Jr, Kakar S, Chung $\mathrm{DC}$, et al. Low rate of microsatellite instability in young patients with adenomas: reassessing the Bethesda guidelines. Am J Gastroenterol. 2005;100:1143-9.

40. Walsh MD, Buchanan DD, Pearson SA, Clendenning M, Jenkins MA, Win AK, et al. Immunohistochemical testing of conventional adenomas for loss of expression of mismatch repair proteins in Lynch syndrome mutation carriers: a case series from the 
Australasian site of the colon cancer family registry. Mod Pathol. 2012;25:722-30.

41. Walsh MD, Buchanan DD, Walters R, Roberts A, Arnold S, McKeone D, et al. Analysis of families with Lynch syndrome complicated by advanced serrated neoplasia: the importance of pathology review and pedigree analysis. Fam Cancer. 2009;8:313-23.

42. Young J, Barker MA, Simms LA, Walsh MD, Biden KG, Buchanan D, et al. Evidence for BRAF mutation and variable levels of microsatellite instability in a syndrome of familial colorectal cancer. Clin Gastroenterol Hepatol. 2005;3:254-63.

43. Yurgelun MB, Goel A, Hornick JL, Sen A, Turgeon DK, Ruffin MTt, et al. Microsatellite instability and DNA mismatch repair protein deficiency in Lynch syndrome colorectal polyps. Cancer Prev Res. 2012;5:574-82.

44. Basterra M, Gomez M, Mercado Mdel R, Irisarri R, Amorena E, Arrospide A. et al. Prevalence of altered mismatch repair protein nuclear expression detected by immunohistochemistry on adenomas with high-grade dysplasia and features associated with this risk in a population-based study. Gastroenterol Hepatol. 2016;39:500-7.

45. Koh DC, Luchtefeld MA, Kim DG, Attal H, Monroe T, Ingersoll $\mathrm{K}$. Microsatellite instability and MLH1 hypermethylation-incidence and significance in colorectal polyps in young patients. Colorectal Dis. 2007;9:521-6.

46. Kushnir VM, Nalbantoglu I, Watson R, Goodwin J, Safar E, Chokshi RV, et al. Advanced colorectal adenomas in patients under 45 years of age are mostly sporadic. Dig Dis Sci. 2014;59:2757-64.

47. Ricciardiello L, Goel A, Mantovani V, Fiorini T, Fossi S, Chang DK, et al. Frequent loss of hMLH1 by promoter hypermethylation leads to microsatellite instability in adenomatous polyps of patients with a single first-degree member affected by colon cancer. Cancer Res. 2003;63:787-92.

48. Strul H, Liberman E, Kariv R, Gartner M, Kazanov D, Keidar A, et al. Prospective assessment of microsatellite instability in gastrointestinal neoplasia in Ashkenazi and non-Ashkenazi Jews. J Med. 2003;34:139-48.

49. Ueda E, Watanabe T, Umetani N, Ishigami H, Sasaki S, Nagawa $\mathrm{H}$. Microsatellite instability of cancers and concomitant adenomas in synchronous multiple colorectal cancer patients. J Exp Clin Cancer Res. 2002;21:149-54.

50. Ahadova A, Gallon R, Gebert J, Ballhausen A, Endris V, Kirchner $\mathrm{M}$, et al. Three molecular pathways model colorectal carcinogenesis in Lynch syndrome. Int J Cancer. 2018;143:139-50.

51. Chang K, Taggart MW, Reyes-Uribe L, Borras E, Riquelme E, Barnett RM, et al. Immune profiling of premalignant lesions in patients with Lynch Syndrome. JAMA Oncol. 2018;4: 1085-92.

52. Lin SH, Raju GS, Huff C, Ye Y, Gu J, Chen JS, et al. The somatic mutation landscape of premalignant colorectal adenoma. Gut. 2018;67:1299-305.

53. Sheridan TB, Fenton H, Lewin MR, Burkart AL, IacobuzioDonahue CA, Frankel WL, et al. Sessile serrated adenomas with low- and high-grade dysplasia and early carcinomas: an immunohistochemical study of serrated lesions "caught in the act". Am J Clin Pathol. 2006;126:564-71.

54. Goverde A, Wagner A, Bruno MJ, Hofstra RMW, Doukas M, van der Weiden MM, et al. Routine molecular analysis for Lynch syndrome among adenomas or colorectal cancer within a national screening program. Gastroenterology. 2018;155:1410-5.

55. Abdel-Rahman WM, Ollikainen M, Kariola R, Jarvinen HJ, Mecklin JP, Nystrom-Lahti M, et al. Comprehensive characterization of HNPCC-related colorectal cancers reveals striking molecular features in families with no germline mismatch repair gene mutations. Oncogene 2005;24:1542-51.

56. Rijcken FE, van der Sluis T, Hollema H, Kleibeuker JH. Hyperplastic polyps in hereditary nonpolyposis colorectal cancer. Am J Gastroenterol. 2003;98:2306-11.

57. Caldes T, Godino J, Sanchez A, Corbacho C, De la Hoya M, Lopez Asenjo J, et al. Immunohistochemistry and microsatellite instability testing for selecting MLH1, MSH2 and MSH6 mutation carriers in hereditary non-polyposis colorectal cancer. Oncol Rep. 2004;12:621-9.

58. Guastadisegni C, Colafranceschi M, Ottini L, Dogliotti E. Microsatellite instability as a marker of prognosis and response to therapy: a meta-analysis of colorectal cancer survival data. Eur J Cancer. 2010;46:2788-98.

59. Sie AS, Mensenkamp AR, Adang EM, Ligtenberg MJ, Hoogerbrugge N. Fourfold increased detection of Lynch syndrome by raising age limit for tumour genetic testing from 50 to 70 years is cost-effective. Ann Oncol. 2014;25:2001-7.

60. Poynter JN, Siegmund KD, Weisenberger DJ, Long TI, Thibodeau SN, Lindor N, et al. Molecular characterization of MSI-H colorectal cancer by MLHI promoter methylation, immunohistochemistry, and mismatch repair germline mutation screening. Cancer epidemiology, biomarkers \& prevention: a publication of the American Association for Cancer Research, cosponsored by the American Society of Preventive. Oncology. 2008;17:3208-15.

61. Rex DK, Ahnen DJ, Baron JA, Batts KP, Burke CA, Burt RW, et al. Serrated lesions of the colorectum: review and recommendations from an expert panel. Am $\mathrm{J}$ Gastroenterol. 2012;107:1315-29.

62. Andersen SH, Lykke E, Folker MB, Bernstein I, Holck S. Sessile serrated polyps of the colorectum are rare in patients with Lynch syndrome and in familial colorectal cancer families. Fam Cancer. 2008;7:157-62.

63. Staffa L, Echterdiek F, Nelius N, Benner A, Werft W, Lahrmann $\mathrm{B}$, et al. Mismatch repair-deficient crypt foci in Lynch syndromemolecular alterations and association with clinical parameters. PLoS ONE. 2015;10:e0121980.

64. Snowsill T, Coelho H, Huxley N, Jones-Hughes T, Briscoe S, Frayling IM, et al. Molecular testing for Lynch syndrome in people with colorectal cancer: systematic reviews and economic evaluation. Health Technol Assess. 2017;21:1-238.

65. Harris C, Amy D. Ethical issues in the conduct and reporting of meta-analysis. Handbook of ethics in quantitative methodology. (Routledge, London, 2011), pp 415-44. 